\title{
Diagnostic imaging of cardiac amyloidosis
}

Ana Martinez-Naharro ${ }^{1}$, A. John Baksi ${ }^{2,3}$, Philip N Hawkins ${ }^{1}$ and Marianna Fontana ${ }^{1}$

${ }^{1}$ National Amyloidosis Centre, Division of Medicine, University College London, Royal Free Hospital, London, UK.

${ }^{2}$ Royal Brompton Hospital, London, UK

${ }^{3}$ National Heart and Lung Institute, Imperial College London, London, UK

Email: m.fontana@ucl.ac.uk 


\begin{abstract}
Systemic amyloidosis encompasses an underdiagnosed, debilitating, progressive but increasingly recognized group of disorders characterized by the extracellular deposition of misfolded proteins in one or more organs. Cardiac amyloid deposition leads to an infiltrative or restrictive cardiomyopathy and is the major driver of prognosis in systemic amyloidosis. In total, $>30$ proteins can form amyloid fibrils, but the two main types of amyloid that infiltrate the heart are monoclonal immunoglobulin light chain amyloidosis or transthyretin amyloidosis. Cardiac amyloidosis can be acquired in older individuals or inherited at a younger age. Given the nonspecific symptoms of these disorders, a high index of suspicion is paramount to make the correct diagnosis, using non-invasive imaging methods such as echocardiography, bone scintigraphy and cardiac magnetic resonance (CMR). In the past 10 years, the use of CMR with tissue characterization and bone scintigraphy to diagnose cardiac amyloidosis has revolutionized our understanding of the disease, leading to changes in patient care. However, there remains a need for better awareness, expertise and greater clinical suspicion, since the initial clues provided by electrocardiography and echocardiography might not be typical. With specific treatments now available, timely diagnosis of cardiac amyloidosis is more important than ever. In this Review, we discuss the current and novel approaches for the diagnosis and treatment of cardiac amyloidosis.
\end{abstract}




\section{Introduction}

Cardiac amyloidosis is considered the paradigm of the restrictive cardiomyopathies.

Previously thought to be very rare, all forms of cardiac amyloidosis are now understood to be underdiagnosed $^{1,2}$. Timely diagnosis of cardiac amyloidosis is critical, given the recent availability of an array of effective new treatments. Many types of amyloidosis can involve the heart, but two types predominate ${ }^{3}$ : immunoglobulin light-chain (AL) amyloidosis and transthyretin (ATTR) amyloidosis. ATTR-related amyloidosis is in turn further classified into a hereditary form (previously known as familial amyloid cardiomyopathy or familial amyloid polyneuropathy depending on the predominant symptoms) that is associated with different mutations in TTR, and the more common non-hereditary wild-type form (historically known as senile systematic or cardiac amyloidosis), which is a late onset disease affecting mostly men. Until very recently, AL amyloidosis was thought to be the most common type of systemic amyloidosis, with an estimated prevalence of 8-12 per million person-year ${ }^{4}$. AL amyloidosis most frequently affects the kidneys and can lead to nephrotic syndrome ${ }^{5}$, whereas cardiac involvement is the second most common presenting manifestation, occurring in $50 \%-75 \%$ of all cases ${ }^{6,7}$. Other organs that might be involved include the peripheral and autonomic nervous system, the vasculature, the liver and gastrointestinal tract, and soft tissues ${ }^{7}$. Symptoms of AL amyloidosis reflect the multisystemic involvement of the disease and are mostly non-specific, such as fatigue, dyspnoea, weight loss, peripheral oedema and signs of autonomic or peripheral neuropathy. Other typical signs, such as periorbital bruising and macroglossia, occur in only a minority of cases ${ }^{5}$. Treatment of AL amyloidosis involves cytotoxic chemotherapeutic agents aimed at suppressing proliferation of the underlying plasma cell clone (and thus inhibits production of the amyloid fibril precursor protein), and in some cases, bone marrow transplantation. AL amyloidosis was considered untreatable until a few years ago, but contemporary therapies have improved patient survival ${ }^{8}$. However, even with standard chemotherapy, patients with AL amyloid cardiomyopathy (AL-CM) and elevated levels of cardiac biomarkers who are not candidates for autologous stem cell transplantation continue to have poor outcomes, with $40 \%$ of patients dying within 2 years of diagnosis and only $20 \%$ of responders experiencing cardiac improvement as assessed by biomarker criteria9 9

ATTR amyloid cardiomyopathy (ATTR-CM) was once considered a rare cause of heart failure, but its clinical significance has been increasingly recognized in the past 10 years ${ }^{10}$. Current reports estimate a prevalence of $10 \%-16 \%$ in some cohorts (especially elderly men), 
and autopsy studies revealed that amyloid deposits derived from plasma transthyretin were present in the hearts of up to $25 \%$ of elderly patients ${ }^{11,12}$. Wild-type ATTR (ATTRwt) almost exclusively affects the heart and only carpal tunnel syndrome (often bilateral) accompanies the cardiac phenotype. The presentation of mutant ATTR (ATTRm) is more varied and depends on the specific mutation, geographical area, ethnicity, age and sex ${ }^{13}$. ATTRm is inherited in an autosomal dominant pattern. To date, $>120$ pathogenic mutations have been identified, however, only a few of these variants are responsible for the majority of cases of ATTRm worldwide, notably Val30Met, Thr60Ala, Ser77Tyr and Val122Ile ${ }^{14,15}$. In some cases of ATTRm, such as those involving a Val30Met mutation (endemic in certain regions of Japan, Portugal or Sweden) peripheral neuropathy or autonomic dysfunction might be the predominate characteristic, with cardiac amyloid either being absent or limited to very early cardiac amyloid infiltration ${ }^{16}$. Other mutations such as Thr60Ala (the most common cause of hereditary ATTR in the UK) often present predominantly with cardiomyopathy. Furthermore, a heterozygous Val122Ile mutation is present in $\sim 4 \%$ of African Americans, which can cause a late-onset cardiomyopathy ${ }^{17,18}$. Genetic testing to identify mutations in TTR should be performed in all patients with ATTR-CM, given the important implications for family members and potential for genetic counselling.

ATTR-CM is associated with a better prognosis than AL-CM, with a survival of typically 4 to 5 years from diagnosis ${ }^{19,20}$. The median survival rate of untreated AL-CM is less than 6 months. Advances in non-invasive diagnosis, coupled with the development of effective therapies $^{21-24}$, have shifted ATTR-CM from a rare and untreatable disease to a condition that clinicians should consider on a daily basis. The diagnosis of amyloidosis remains challenging and relies on a high index of clinical suspicion (Box 1). Unfortunately, the disease is frequently asymptomatic until the late stage and can present with highly variable or nonspecific symptoms. Approximately $40 \%$ of patients are misdiagnosed and it takes on average 6 months and visits to three doctors prior to a correct diagnosis of ATTR-CM ${ }^{25}$.

\section{[H1] Clinical features and biomarkers}

Cardiac amyloidosis typically manifests with signs and symptoms of heart failure and poor exercise tolerance owing to low cardiac output ${ }^{26}$. These symptoms are often accompanied by hypotension, which complicates heart failure management. Furthermore, patients with cardiac amyloidosis frequently present with syncope (often exertional), reflecting the limited capacity of the heart to increase diastolic filling. Syncope can also be aggravated by antihypertensive 
medications and concomitant autonomic neuropathy can precipitate orthostatic hypotension. Conduction system disease is more common in patients with ATTR-CM than in AL-CM, in particular those with ATTRwt, whereby up to one-third of patients with ATTRwt require permanent pacemakers ${ }^{27}$. Atrial fibrillation alongside a controlled ventricular response is often present in these patients owing to the underlying conduction disease; when present, atrial fibrillations becomes persistent in most patients with ATTRwt ${ }^{28}$. Patients with cardiac amyloidosis have increased risk of intracardiac thrombus, which might even occur in sinus rhythm ${ }^{29,30}$. Some patients will also present with stroke or systemic embolization, usually because of unrecognized atrial fibrillation.

In our experience, generally ATTR-CM is characterized by 2 to 3 years of relatively stability despite advanced disease on imaging, followed by deterioration to severe and refractory heart failure, suggesting that disease progression is slow. By contrast, AL-CM is characterized by subtle changes in structure as shown on imaging, despite rapidly progressive heart failure symptoms ${ }^{19}$. The discrepancy in imaging results between the two types of cardiac amyloidosis, especially wall thickness and clinical course, highlights the fact that cardiac amyloidosis is not a simple infiltrative disorder ${ }^{7}$.

Cardiac biomarkers might be helpful for raising clinical suspicion of cardiac amyloidosis in patients with a known plasma cell dyscrasia (heterogeneous group of disorders caused by the monoclonal proliferation of lymphoplasmacytic cells in the bone marrow) or suspected ATTR-CM, and should prompt further investigations. The combination of serum N-terminal pro-B-type natriuretic peptide (NT-proBNP), troponin and renal function is useful for risk stratification $^{31,32}$ and can help guide treatment strategies ${ }^{33}$.

\section{[H1] Diagnostic techniques}

\section{[H2] Electrocardiography}

Electrocardiography has an important role in raising diagnostic suspicion of cardiac amyloidosis. Electrocardiographic assessment of voltage in patients with cardiac amyloidosis is not influenced by the same factors as in the general population. Classic predictors of voltage such as age, sex, ethnicity, blood pressure levels, body surface area and smoking status are not associated with voltage that is measured using limb and precordial (Sokolow) voltage criteria ${ }^{34}$. In addition, the established linear relationship between left ventricular (LV) 
mass and ECG voltage does not hold true for cardiac amyloidosis, given that ECG is abnormal in almost all patients with this disease ${ }^{35,36}$. The classic hallmark of the disease has been described as the combination of low QRS voltage on ECG and increased LV wall thickness on echocardiogram ${ }^{37,38}$. Because the thickening of the ventricle in amyloidosis is due to myocardial infiltration rather than cardiomyocyte hypertrophy, the ECG limb lead voltage tends to decrease as the ventricle thickens and is often associated with extreme leftaxis or right-axis deviation. However, only $~ 50 \%$ of patients with AL-CM and about $25 \%$ $40 \%$ of patients with ATTR-CM meet true low-voltage criteria (that is, QRS amplitude $<5$ $\mathrm{mm}$ in limb leads or $<10 \mathrm{~mm}$ in precordial leads ${ }^{19,39,40}$ ). Hence, the absence of low-voltage criteria does not exclude the diagnosis of cardiac amyloidosis. Although voltage criteria for LV hypertrophy are extremely uncommon in patients with AL-CM, they can be present in up to quarter of patients with ATTR-CM ${ }^{41}$. A possible explanation for the discrepancy in QRS voltages between these two subtypes of cardiac amyloidosis is that ATTR-CM is associated with a greater relative increase in cardiomyocyte hypertrophy, and thus higher QRS voltages, than AL-CM ${ }^{42,43}$.

Another main feature of ECG associated with cardiac amyloidosis is the presence of a pseudoinfarct pattern with Q waves in the precordial or limb leads mimicking a prior anteroseptal, inferior or lateral myocardial infarction ${ }^{40,44}$. This finding is seen in $\sim 50 \%$ of patients $^{44}$. Furthermore, atrioventricular (AV) heart block, particularly second-degree and third-degree AV block, are also common and often necessitate pacemaker implantation in patients with cardiac amyloidosis, but sinus node dysfunction has also been observed. Firstdegree AV block has been reported in 56\% of patients with ATTR-CM associated with the Val122Ile variant ${ }^{41}$.

Other common features present in the ECGs of patients with cardiac amyloidosis include left anterior hemiblock, ischaemic or nonspecific $\mathrm{T}$ wave abnormalities, and rhythm disturbances, particularly atrial fibrillation, which has been reported in up to $70 \%$ of patients with ATTRwt amyloidsis $^{28}$. Ventricular arrhythmias are also common, although the first clinically apparent evidence of an abnormal ventricular rhythm might be ventricular tachycardia or fibrillation in the setting of a non-resuscitable cardiac arrest.

\section{[H2] Echocardiography}

Amyloid deposits can accumulate in cardiac chambers, vessels and valves, but the infiltrative process is most marked in the ventricular walls, which results in thickened (most commonly 
symmetric in AL-CM, but asymmetric in ATTR-CM ${ }^{45}$ ), non-dilated ventricles. The subsequent elevation of pressure in the atria is associated with mild atrial dilatation, as the thickening of the atrial walls by amyloid deposition prevents severe dilatation.

Echocardiography is a valuable and widely accessible tool for investigating heart failure, and although it often provides the first clues to the presence of cardiac amyloidosis, it is neither sensitive nor specific for this disorder ${ }^{46}$. Typical echocardiographic findings include thickening of ventricular walls, small LV chamber volume, valve thickening, atrial enlargement and signs of elevated filling pressures such as pericardial and pleural effusions and dilated vena cava owing to restrictive diastolic filling. A 'granular sparkling' appearance of the myocardium has been traditionally described as a typical sign, but over time has been proven to be a non-specific presentation ${ }^{37}$. Furthermore, an interventricular septal thickness $>12 \mathrm{~mm}$ in the absence of aortic valve disease or substantial systemic hypertension remains a key echocardiographic feature that is indicative of cardiac involvement in patients with systemic AL amyloidosis ${ }^{47}$.

Ejection fraction is typically preserved early in the disease process ${ }^{48,49}$, but LV performance deteriorates with disease progression ${ }^{50}$. Cardiac amyloidosis characteristically presents as a continuum of diastolic dysfunction that progresses from impaired relaxation to a pseudonormal pattern to a restrictive pattern involving increased deposition of amyloid in the myocardium ${ }^{51}$. Stroke volume index and myocardial contraction fraction (ratio of stroke volume to myocardial mass) have been shown to be better diagnostic markers of cardiac amyloidosis than ejection fraction ${ }^{49}$. In addition, reduction in peak systolic wall motion velocities, which disproportionally affect the longitudinal rather than the radial axes, present early in the course of the disease ${ }^{49}$. Reduced ejection fraction at diagnosis is more common in patients with ATTR-CM with the Val122Ile variant than those with ATTRwt ${ }^{38}$, which likely reflects a more advanced stage of disease at diagnosis and perhaps accounts for the reduced survival reported in these patients ${ }^{20}$.

The majority of conventional echocardiographic parameters have low accuracy for diagnosing cardiac amyloidosis, mostly owing to low sensitivity ${ }^{45}$. However, several echocardiographic indices have high specificity, especially $\mathrm{E} / \mathrm{E}^{\prime}$ ratio (when $>9.6$ has a sensitivity of $50 \%$ and a specificity of $100 \%$ ), left atrial volume index (when $\geq 47 \mathrm{ml} / \mathrm{m}^{2}$ has a sensitivity of $44 \%$ and a specificity of $93 \%$ ) and myocardial contraction fraction (when $\leq 0.234$ has a sensitivity of $56 \%$ and a specificity of $96 \%)^{52}$ and, therefore, might be useful for 
potential amyloidosis cases ${ }^{53}$. Among the conventional echocardiographic parameters, myocardial contraction fraction has shown the best diagnostic accuracy, with an area under the curve (AUC) of $0.80^{52}$.

Longitudinal strain measured by tissue Doppler imaging and echocardiographic speckle tracking has emerged as a useful clinical marker of cardiac amyloidosis, which can help to distinguish the disease from other causes of wall thickening such as hypertension and hypertrophic cardiomyopathy. A longitudinal strain gradient showing relative preservation of function at the apex and significant impairment of the mid and basal segments is a very consistent and characteristic finding in patients with cardiac amyloidsis ${ }^{54,55}$. This phenomenon gives rise to a distinctive 'bulls-eye' pattern when the segmental strain is plotted, which is rarely seen in other cardiomyopathies (Figure 1). Despite the extensive literature on the apical sparing of longitudinal strain in amyloidosis ${ }^{37,54,56}$, the pathophysiological mechanism underlying this phenomenon remains unclear. Several mechanisms have been proposed, including the presence of less amyloid deposition at the apex compared with the base, the diversity in myocardial fibre orientation at the apex, and greater tendency towards apoptosis and remodelling in the basal segments related to higher parietal stress and turbulent flow ${ }^{57}$. Furthermore, deformation-based parameters such as longitudinal strain have higher sensitivities and specificities for the detection of ATTR-CM Global longitudinal strain $\geq-15.1$ has a sensitivity of $87 \%$ and a specificity of $72 \%$ for diagnosis of ATTR-CM, with an AUC of $0.85^{52}$.

Finally, many structural and functional differences exist between AL-CM and ATTR-CM. AL-CM is associated with only slightly increased wall thickness, but more haemodynamic derangement compared with ATTR-CM (especially cardiomyopathy associated with ATTRwt ${ }^{19}$, whereas ATTR-CM is characterized by a greater degree of increase in LV and right ventricular (RV) mass, and more systolic dysfunction. The discrepancy between the two types of amyloid cardiomyopathy in imaging findings and clinical course highlights the fact that AL-CM is not a simple infiltrative disorder, and should be more accurately characterized as a 'toxic-infiltrative' cardiomyopathy ${ }^{7}$. Given the high degree of overlap between the echocardiographic features cardiac amyloidosis and other cardiomyopathies, echocardiography alone cannot be used to differentiate the many different pathologies associated with increased wall thickness, but should prompt a low threshold for further multimodality assessment. 


\section{[H2] Cardiovascular magnetic resonance}

In the past decade, CMR has emerged as a robust imaging technique that not only provides structural and functional data, but most importantly conveys valuable information regarding tissue composition. An exponential increase has been observed in the use of CMR to assess the hearts of patients with systemic amyloidosis, with approximately $85 \%$ of all publications on this subject arising in the past 10 years alone ${ }^{58}$. This increase has been largely driven by the emergence of new CMR sequences and their subsequent modernisation and technical development, which has led to an increased awareness and recognition of the cardiac amyloidosis.

$\mathrm{CMR}$ can produce high resolution and 3D images of the heart, and has many advantages over other traditional imaging techniques in that it does not depend on geometry, and consistently provides excellent delineation of the endocardium and epicardium ${ }^{59}$. Cardiac amyloidosis was historically thought to be characterised by concentric and symmetric hypertrophy of the left ventricle. However, CMR has since revealed that the most common morphological phenotype of patients with ATTR-CM is asymmetric LV hypertrophy, which is present in $79 \%$ of these patients ${ }^{60}$. The pattern of asymmetrical septal hypertrophy can be divided into the morphological subtypes of sigmoid septum (present in 55\% of patients with ATTR-CM) and reverse septal contour (in $24 \%$ of patients with ATTR-CM). Notably, no differences in morphological phenotype were observed between the ATTRwt and ATTRm subtypes. Symmetrical and concentric LV hypertrophy was present in only $18 \%$ of patients with ATTR-CM, but was present in $68 \%$ of patients with AL-CM, and the most common form of ventricular remodelling ${ }^{60}$. Interestingly, the asymmetrical pattern with reverse septal contour was not observed in patients with AL-CM, an important finding given that the association between cardiac amyloidosis and concentric hypertrophy has an important role in the misdiagnosis of ATTR-CM. For example, up to $6 \%$ of patients diagnosed with hypertrophic cardiomyopathy actually have ATTR-CM ${ }^{38}$.

Importantly, CMR can accurately characterize myocardial tissue based on the intrinsic magnetic properties of different tissues ( $\mathrm{T} 1, \mathrm{~T} 2$ and $\mathrm{T} 2 *)$ without the use of gadoliniumbased contrast agents. However, these intrinsic properties can be accentuated by administration of gadolinium-based contrast as in the late gadolinium enhancement (LGE) technique and post-contrast T1 for extracellular volume (ECV) calculation ${ }^{61}$. Gadoliniumbased contrast agents accelerate the relaxation of the water molecules present in tissues to 
give rise to an enhanced signal on T1-weighted images and, together with appropriate sequence parameters, an improved image contrast ${ }^{62}$. Gadolinium chelates are extracellular contrast agents that cannot cross the intact cardiomyocyte cell membrane ${ }^{63}$. In the normal myocardium, cardiomyocytes are densely packed and cardiomyocyte intracellular space forms the majority ( $\sim 85 \%)$ of the myocardial volume ${ }^{64}$. In cardiac amyloidosis, the extracellular space expands owing to increased amyloid deposition, which leads to elevated gadolinium concentration in the myocardium and thus hyperenhancement ${ }^{65}$.

LGE imaging provides pathognomonic findings with high diagnostic accuracy for cardiac amyloidosis ${ }^{66,67}$. Amyloid cardiomyopathy presents initially as a typical pattern of diffuse subendocardial LGE that can become transmural in the later stages of the disease ${ }^{67}$. This pattern is coupled with abnormal gadolinium kinetics with the myocardium and blood nulling at the same time or the myocardium nulling before the blood ${ }^{66,68}$. However, caution should be taken when using protein-bound contrast agents for myocardial enhancement in nonischaemic cardiomyopathy, since diagnostic performance might not be the same as for non-protein-bound variants ${ }^{69}$. Protein-bound contrast agents are partially 'intravascular', and thus concerns have been raised for its use in quantification of $\mathrm{ECV}^{70}$.

Traditional LGE imaging is a thresholding or comparison technique, the operator choses the null inversion time (TI) according to what is considered normal myocardium. By convention, areas with the most contrast should be displayed as bright and normal myocardium should be displayed as black on LGE imaging. The accuracy of a chosen null TI in a clinical setting depends on operator expertise, clearance rate of the contrast agent and patient tolerance to additional breath-hold acquisitions ${ }^{71}$, which all can vary widely in clinical practice. Nulling the normal myocardium in cardiac amyloidosis might be very challenging since infiltration is frequently diffuse throughout the myocardium and areas of normal myocardium might not be available for comparison; for example, the operator might erroneously choose to null the abnormal myocardium that is missing global infiltration ${ }^{72}$. Newer techniques, particularly phase-sensitive inversion recovery (PSIR), an LGE image reconstruction technique that is less sensitive to operator choice of null point and can render signal intensity truly T1weighted, might be more accurate in determining the extent of cardiac involvement ${ }^{71}$. Furthermore, PSIR is also easier for the operator than traditional LGE imaging (as less precision in setting the TI is needed) and is available from all CMR manufacturers. 
Three LGE patterns are widely recognised in patients with cardiac amyloidosis: no LGE, subendocardial LGE and transmural $\mathrm{LGE}^{72}$, all of which show good correlation with the degree of myocardial infiltration ${ }^{60}$. However, LGE imaging has several limitations, particularly in patients with cardiac amyloidosis, many of whom have renal impairment and cannot tolerate gadolinium-based contrast agents ${ }^{73,74}$. T1 mapping can quantitatively measure the progression of cardiac amyloid infiltration from early infiltration without LGE to massive diffuse transmural involvement ${ }^{43}$. T1 mapping, before administration of contrast ${ }^{75,76}$, can measure the intrinsic signal from the myocardium, (known as native myocardial T1). Coupled with studies performed after administration of the gadolinium-based contrast, T1 mapping can be used to calculate myocardial ECV — that is, how much of the extracellular space is occupied by amyloid deposits. Native T1 and ECV are elevated in both AL-CM and ATTR-CM $43,77,78$ and have both been extensively validated as indicators of cardiac amyloid infiltration ${ }^{43,66,79}$, correlating with infiltration measured by other techniques such as ${ }^{99 \mathrm{~m}} \mathrm{Tc}-3,3-$ diphosphono1,2-propanodicarboxylic acid $\left({ }^{99 \mathrm{~m}} \mathrm{Tc}-\mathrm{DPD}\right)$ scintigraphy ${ }^{43,76}$. Furthermore, in a single centre study, native myocardial T1 elevation was associated with high diagnostic accuracy for cardiac amyloidosis when the pretest probability was high ${ }^{78}$, whereby myocardial $\mathrm{T} 1$ was increased before the onset of LV hypertrophy, evidence of LGE or increase in blood biomarkers ${ }^{75}$. A clinical algorithm using native myocardial T1 has been developed to enable the diagnosis of cardiac amyloidosis without the need for gadolinium-based contrast agents in a large proportion of patients with suspected cardiac amyloidosis ${ }^{78}$. Most importantly, the use of native $\mathrm{T} 1$ can also be used in patients with severe renal disease, a common comorbidity in those with cardiac amyloidsis ${ }^{78}$.

Although native T1 and ECV were thought to be similar in the two main subtypes of cardiac amyloidosis, native $\mathrm{T} 1$ has since been found to be higher in those with AL-CM, whereas ECV is greater in those with ATTR-CM ${ }^{43,80}$. These differences probably reflect different biological data provided by native T1 and ECV measurements. Native T1 measures the composite myocardial signal from the interstitium and cardiomyocytes without differentiating between the underlying processes. Cardiac amyloidosis is emerging as a condition characterized by variable degrees of amyloid infiltration, myocardial oedema and differential cardiomyocyte response, often with cardiomyocyte hypertrophy. Contrast administration and ECV measurements enable the isolation of the signal from the extracellular space, but native myocardial T1 provides a composite signal from the intracellular and extracellular spaces that is potentially influenced by other pathophysiological mechanisms beyond simple amyloid 
load. Native myocardial T1 is highly influenced by water content in the tissue, and therefore, would be elevated in the presence of myocardial oedema ${ }^{81}$. On the other hand, an increase in cardiomyocyte hypertrophy (as often seen in patients with ATTR-CM) likely results in a reduction in native $\mathrm{T} 1^{80}$. Consequently, $\mathrm{ECV}$ is a much more robust marker of true amyloid infiltration ${ }^{43}$. ECV is also elevated during early cardiac infiltration before LGE is present, and conventional clinical testing has detected cardiac involvement in patients with high pre-test probability, suggesting that ECV is marker of early disease ${ }^{82}$. Furthermore, ECV correlates with markers of disease severity, such as cardiac function, blood biomarkers and functional status. Both native T1 and ECV are predictors of prognosis in patients with AL-CM ${ }^{79}$ and ATTR-CM ${ }^{43}$, but only ECV remains an independent predictor of prognosis for ATTR-CM after adjusting for known predictors. ECV is also the earliest marker of cardiac amyloid regression after successful therapy in patients with AL amyloidosis ${ }^{83}$.

Another intrinsic property of the myocardium that can be measured with CMR is T2. High signal on T2 imaging of the heart is indicative of myocardial oedema, typically seen in acute myocarditis, infarction or inflammatory cardiomyopathies such as cardiac sarcoidosis. T2 has been shown to be elevated in both AL and ATTR amyloidosis, with the greatest elevation of $\mathrm{T} 2$ present in patients with AL amyloidosis before starting chemotherapy ${ }^{84}$. T2 is also a predictor of mortality in AL amyloidosis, lending support to an independent role of myocardial oedema on outcomes in these patients.

CMR offers extensive structural and functional data, which, when coupled with LGE imaging and mapping (native T1, T2, ECV), permits understanding of the different processes underlying the progression of cardiac amyloidosis. These processes include pure amyloid infiltration (assessed by amyloid burden and ECV), myocardial oedema (with T2 being the most specific marker), cardiomyocyte response (calculated using LV mass and ECV) ${ }^{80}$ and disease severity (graded from ECV elevation to no LGE, subendocardial LGE or transmural LGE). Although native T1 and T2 values tend to be higher in patients with AL-CM (particularly in untreated patients ${ }^{84}$ ) whereas ECV is higher in patients with ATTR-CM, none of these CMR techniques can be used to definitively differentiate between the two types of cardiac amyloidosis in an individual patient.

Finally, myocardial perfusion can also be measured by CMR with fully automated myocardial blood flow mapping ${ }^{85}$. Intramyocardial vessels are frequently infiltrated by amyloid, resulting in impaired vasodilatation, which can cause global myocardial 
ischaemia ${ }^{86}$. Cardiac biomarkers such as troponin T and NT-proBNP are known to be constantly elevated in patients with cardiac amyloidosis ${ }^{87}$. Myocardial perfusion has been proposed to be reduced at rest in patients with cardiac amyloidosis ${ }^{88}$, which might contribute to increased levels of cardiac biomarkers have an important role in the response to new amyloid therapies that directly target amyloid deposits. (Figure 2).

\section{[H2] Radionuclide bone scintigraphy}

The first nuclear imaging studies using bone-seeking agents in cardiac amyloidosis were performed as early as the $1980 \mathrm{~s}^{89}$, but it was not until 20 years later that Puille and colleagues described the potential use of bone scintigraphy to identify amyloid deposits in ATTR-CM ${ }^{90}$. Quantification of the intensity of radiotracer uptake is key in the diagnosis of ATTR-CM using bone scintigraphy. The intensity of retention of bone-avid radiotracers in the heart can be interpreted by semi-quantitative visual analysis, by grading myocardial uptake to rib uptake on planar or single-photon emission computerized tomography (SPECT) imaging, and by quantifying radiotracer uptake using a heart-to-contralateral lung $(\mathrm{H} / \mathrm{CL})$ ratio $^{91}$. The current diagnostic criteria for patients with ATTR-CM include visual myocardial uptake equal or greater than that in bone (specifically in the ribs) or a $\mathrm{H} / \mathrm{CL}$ ratio $\geq 1.5^{92}$. An H/CL ratio of $\geq 1.6$ is associated with poor survival ${ }^{93}$.

Perugini and colleagues have classified cardiac amyloid uptake based on a simple visual scoring system of the delayed $(3 \mathrm{~h}$ ) planar image, in which grade of 0 means no cardiac uptake, a grade of 1 means mild cardiac uptake (less than in bone), a grade of 2 means cardiac uptake greater than bone (but uptake in bone remains clearly visible) and a grade of 3 is indicative of substantial cardiac uptake with a weak or no signal evident in bone ${ }^{94}$ (Figure 5). Although the basis for localisation of radioactive bone tracers to cardiac amyloid remains unknown, the technique has been validated and seems to be sufficiently sensitive to detect early cardiac ATTR amyloid deposits in asymptomatic individuals in whom echocardiography and gadolinium-enhanced CMR images are normal ${ }^{95}$.

Over the past few decades, different bone tracers, including ${ }^{99 \mathrm{~m}} \mathrm{Tc}-$ pyrophosphate $\left({ }^{99 \mathrm{~m}} \mathrm{Tc}-\right.$ PYP) $)^{93,96,97}$, ${ }^{99 \mathrm{~m}} \mathrm{Tc}$-hydroxymethylene diphosphonate ( $\left.{ }^{99 \mathrm{~m}} \mathrm{Tc}-\mathrm{HMDP}\right){ }^{97,98}$ and ${ }^{99 \mathrm{~m}} \mathrm{Tc}-$ DPD $^{90,94,95}$ have been used to diagnose cardiac amyloidosis with similar diagnostic performance ${ }^{99}$. Importantly, however, not all bone tracers are suitable for diagnosing this condition. For example, the widely available bone tracer ${ }^{99 \mathrm{~m}} \mathrm{Tc}-\mathrm{methylene}$ diphosphonate is considered inappropriate for the evaluation of patients with suspected ATTR-CM, given its 
low sensitivity ${ }^{99}$. The mechanism underlying the myocardial retention of the different radiotracers remains unknown but has been attributed to the presence of microcalcifications that are more common in ATTR than in AL cardiac tissue ${ }^{100,101}$.

Bone scintigraphy with a Perugini grade of 2 or 3 describing myocardial uptake showed a high sensitivity of $>99 \%$ for ATTR-CM but a lower specificity of $82-86 \%$, since grade of 1 or 2 can be observed in patients with AL-CM ${ }^{102}$. However, if urine and serum tests are negative for AL amyloidosis, the specificity of the test increases to $100 \%$. Mild uptake of amyloid (grade 1) can also be noted in other subtypes of cardiac amyloidosis such as serum amyloid $\mathrm{A}$ and apolipoprotein $\mathrm{A} 1^{103}$. These findings from studies in the past 5 years have changed the diagnostic pathway of patients with cardiac amyloidosis, such that only a minority of patients with ATTR-CM require endomyocardial biopsies. In the absence of histological data, ATTR-CM can be diagnosed with confidence when a patient presents with clinical phenotype that is associated with an echocardiogram or CMR consistent with amyloidosis, grade 2 or 3 tracer uptake in the heart on radionuclide bone scintigraphy and absence of detectable monoclonal immunoglobulin in the blood and urine using sensitive assays. Histological confirmation and typing of amyloid should be pursued in patients who fail to meet all these criteria, most notably those in whom a monoclonal immunoglobulin is detected, which raises the suspicion of AL amyloidosis ${ }^{104}$. Thereafter, transthyretin genotyping is pertinent to distinguish between ATTRwt and ATTRm.

However, ATTR-CM associated with the Se77Tyr variant has been reported to present with an atypical appearance on bone scintigraphy with only grade 1 uptake, despite having typical clinical, morphological and functional features on echocardiography and CMR (typical LGE imaging and elevated ECV), and expected increases in cardiac biomarkers ${ }^{60}$. This observation suggests that patients with ATTR-CM associated with the Se77Tyr variant have less DPD uptake than expected, given the amyloid burden. Furthermore, ATTR-CM associated with rare mutations in which amyloid deposits mainly consist of full-length transthyretin show no or minimal cardiac uptake of bone-seeking tracers.

In addition to its high sensitivity and specificity, quantitative assessment of bone tracer uptake also provides prognostic information. Increased myocardial retention of the different bone tracers is associated with major adverse cardiac events, acute heart failure and increased mortality ${ }^{92,95,97}$. However, the comparative performance of the different tracers remains 
unclear as ${ }^{99 \mathrm{~m}} \mathrm{Tc}-\mathrm{PYP}$ is most commonly used in the USA, ${ }^{99 \mathrm{~m}} \mathrm{Tc}-\mathrm{HMDP}$ in France and ${ }^{99 m}$ Tc-DPD in other countries such as the UK and Italy. The importance of low-grade cardiac uptake (in particular grade 1) is also unclear. With widespread availability of expensive new therapies, these questions will become important points to consider around cost-benefit.

\section{Positron emission tomography}

Positron emission tomography (PET) is emerging as a useful diagnostic tool for cardiac amyloidosis. Several PET tracers such as ${ }^{18} \mathrm{~F}$-florbetapir, ${ }^{18} \mathrm{~F}$-florbetaben, ${ }^{18} \mathrm{~F}$-flutemetamol and ${ }^{11} \mathrm{C}$-Pittsburgh $\mathrm{B}(\mathrm{C}-\mathrm{PiB})$ have been used successfully to diagnose cardiac amyloidosis ${ }^{105}$. These tracers have been shown to bind specifically to brain $\beta$-amyloid plaques, allowing diagnosis and follow-up of patients with Alzheimer's disease ${ }^{106}$. These tracers likely bind to the $\beta$-pleated structure of amyloid fibril, which facilitates the identification of amyloid deposits independently of the precursor protein. PET tracers are quantitative tools, permitting the measurement of amyloid burden. Small studies have demonstrated that ${ }^{18} \mathrm{~F}$-florbetapir is taken up in the heart of patients with cardiac amyloidosis, with a trend towards a higher myocardial retention index in patients with AL-CM versus those ATTR-CM, and no significant uptake in the healthy controls ${ }^{107,108}$. Similar findings have been observed with ${ }^{11} \mathrm{C}$ $\mathrm{PiB}^{109,110}$ and ${ }^{18} \mathrm{~F}$-florbetaben ${ }^{111}$. In another small study ${ }^{18} \mathrm{~F}$-florbetaben was evaluated in patients with AL-CM $(n=5)$ or ATTR-CM $(n=5)$ and compared with control patients with hypertension $(n=4)$. Myocardial retention was higher in patients with AL-CM and ATTRCM compared with controls, and myocardial retention inversely correlated with LV global and RV free wall longitudinal strain ${ }^{111}$. However, an onsite cyclotron is required for the production of ${ }^{11} \mathrm{C}$ tracers, given their short half-life of 20 mins. The longer half-life of ${ }^{18} \mathrm{~F}$ tracers (110 min) means that it can be distributed and used for research and clinical applications at sites without a cyclotron, making its use more practical compared with ${ }^{11} \mathrm{C}$ - 
PiB tracers. ${ }^{18} \mathrm{~F}$-flutemetamol is an ${ }^{18} \mathrm{~F}$ structural analogue of ${ }^{11} \mathrm{C}-\mathrm{PiB}$ with a benefit of a longer half-life, but this tracer has not yet been studied in the setting of cardiac amyloidosis.

Overall, these promising agents allow absolute quantification of amyloid burden, but are still early in development. Further studies are required to assess their capacity to detect early disease and monitor treatment response. At present, a lack of robust data and the prohibitive costs of these molecules limit its use in the clinic.

\section{[H1] Integration of diagnostic techniques}

All the cardiac imaging techniques described above need to be interpreted alongside clinical findings, which can vary from patient to patient. These imaging techniques are not just useful for the diagnosis of cardiac amyloidosis, but can help to identify the amyloid type, estimate disease severity, track disease progression and monitor treatment response. An approach that integrates all imaging modalities with biomarker testing and tissue biopsy is key for the noninvasive diagnosis of suspected cardiac amyloidosis ${ }^{104}$. Table 1 summarizes the benefits of the techniques most commonly used at each stage of the diagnostic process in cardiac amyloidosis.

\section{[H2] Diagnosis in specific populations}

Certain populations benefit from exclusion or confirmation of cardiac amyloidosis as a differential diagnosis. Echocardiography is invariably the initial imaging modality used to assess all patients with cardiac symptoms and suspect cardiac amyloidosis, although echocardiographic data can change the pretest probability, which in many cases remains equivocal with nonspecific findings.

[H3] Elderly patients with unexplained heart failure and preserved ejection fraction. Heart failure with preserved ejection fraction (HFpEF) currently accounts for up to half of all cases of heart failure ${ }^{12,113}$. Importantly, ATTRwt has been proposed as an underdiagnosed disease that accounts for up to $13 \%$ of all cases of $\mathrm{HFpEF}^{114}$. Although overlap between the clinical presentation of ATTRwt and HFpEF is high, several characteristics such as higher biomarker levels in the blood, increased LV mass, the presence of pericardial effusion and lower voltage-to-mass ratio in ECG are clues that should increase the suspicion of ATTRwt among patients with HFpEF. If cardiac amyloidosis (presumably ATTRwt) is suspected in an elderly patient with HFpEF, bone scintigraphy should be performed. The presence of grade 2 or 3 cardiac uptake might indicate a diagnosis of ATTRwt. The presence of plasma cell dyscrasia 
in blood or urine should be excluded (to exclude AL-CM) and gene sequencing should also be performed as the diagnosis of ATTRm has familial implications.

[H3] Aortic stenosis. ATTR-CM associated with ATTRwt and calcific aortic stenosis are more often seen in elderly individuals. ATTR-CM has a prevalence of $6 \%-12 \%$ in patients with severe aortic stenosis undergoing valve replacement ${ }^{115,116}$. The coexistence of these two conditions has several important clinical implications on diagnosis, management and prognosis $^{117}$. Among 151 consecutive patients aged $>65$ years referred for transcatheter aortic valve replacement (TAVI), ${ }^{99 \mathrm{~m}} \mathrm{Tc}-\mathrm{PYP}$ imaging showed that $16 \%$ showed cardiac uptake consistent with ATTR-CM and 62\% met criteria for low-flow, low-gradient severe aortic stenosis ${ }^{118}$. ATTR-CM should also be considered when assessing prognosis in patients with aortic stenosis, particularly in elderly patients who have received TAVI and those with lowflow, low-gradient aortic stenosis, whereby bone scintigraphy could be routinely used as a screening tool.

[H3] Left ventricular hypertrophy. If echocardiographic results raise the suspicion of cardiac amyloidosis, CMR should be considered if both AL-CM and ATTR-CM or another underlying cause of myocardial hypertrophy (such as hypertrophic cardiomyopathy, hypertensive heart disease or Anderson-Fabry disease) are within the differential diagnosis (Figure 3). CMR has good sensitivity for both types of cardiac amyloidosis ${ }^{60,72}$ and can also identify other common causes of LV hypertrophy ${ }^{119}$. If the CMR findings are indicative of cardiac amyloidosis, serum-free light chains, serum and urine immunofixation and bone scintigraphy should be considered to differentiate between AL and ATTR amyloidosis. Grade 2 or 3 bone scintigraphy coupled with no evidence of a plasma cell dyscrasia in blood or urine is highly specific for ATTR-CM ${ }^{104}$.

\section{[H3] African-Americans individuals aged $>60$ with unexplained heart failure and $L V$}

hypertrophy. The clinical presentation of African-American individuals with heart failure often includes salt-sensitive hypertension, diabetes mellitus and LV hypertrophy ${ }^{120}$. Cardiac amyloidosis, in particular ATTRm associated with the Val122Ile variant, might go undiagnosed when a clinician attributes ventricular hypertrophy or heart failure to other prevalent pathologies such as hypertension, diabetes and obesity. Considering the heterogeneous presentation and relentless progression of this disease, diagnosis is often delayed until the late stages. Bone scintigraphy and CMR should be considered to confirm the diagnosis of cardiac amyloidosis, whereby CMR can also identify other common or rare 
phenocopies of cardiac amyloidosis. To make the diagnosis of ATTR-CM, plasma cell dyscrasia in blood or urine has to be ruled out since AL-CM is a possible, but unlikely diagnosis. Genetic sequencing of TTR should be performed to confirm the Val122Ile mutation.

[H3] Cardiac involvement in systemic AL amyloidosis. If AL-CM is suspected (evidence of plasma cell dyscrasia with suggestive findings on echocardiography or evidence of AL amyloidosis on extra-cardiac biopsy), CMR is the modality of choice to confirm cardiac involvement with high specificity and sensitivity ${ }^{66,121-124}$. CMR can also detect early cardiac infiltration in AL amyloidosis, being positive before hypertrophy and can sometimes identify atypical manifestations such as ventricular dilatation.

\section{[H3] Cardiac involvement in individuals with known or suspected hereditary amyloidosis.}

Bone scintigraphy and/or CMR should be considered in asymptomatic patients with ATTRm or patients with ATTRm with associated polyneuropathy. However, the data for these patients groups are limited and further studies are required to confirm the sensitivity and specificity of the tests.

[H3] Patients with previous carpal tunnel syndrome. Patients with ATTR-CM have been reported to present with symptoms of carpal tunnel syndrome approximately 5-15 years prior to the onset of cardiac symptoms. Carpal tunnel syndrome has a reported prevalence of $39 \%$ $46 \%$ in patients with ATTR-CM ${ }^{32,125}$. A small study revealed that among 98 patients undergoing carpal tunnel release surgery, amyloid deposits were found in tenosynovial tissue in 10 patients, 2 of whom were found to have cardiac involvement ${ }^{126}$. A 2019 study reported that carpal tunnel syndrome is associated with a 12-fold increased risk of amyloidosis, but that the absolute incidence of diagnosed amyloidosis is low ${ }^{127}$. Therefore, carpal tunnel syndrome might not merit direct referral for further evaluation for amyloidosis, but could rather be considered as a red flag and an opportunity for early disease detection ${ }^{127}$.

\section{[H2] Prognostic insights from imaging}

Cardiac amyloidosis is associated with poor survival and as such, many variables can predict prognosis in both AL-CM and ATTR-CM. These prognostic variables can be measured using various imaging techniques, which are complemented by blood biomarkers NT-proBNP and troponin, which form the basis of the Mayo Clinic classification of AL-CM ${ }^{128}$, and also NT- 
proBNP and estimated glomerular filtration rate, used by the National Amyloidosis Centre staging system in ATTR-CM ${ }^{20}$.

[H3] Prognostic data from cardiac structure and function. Many echocardiographic parameters have been associated with outcomes in cardiac amyloidosis, from measures of systolic and diastolic function ${ }^{48,129,130}$ to more advanced strain parameters ${ }^{131,132}$. As markers of systolic function, tricuspid annular plane systolic excursion and stroke volume have been demonstrated to be strong predictors of prognosis by different imaging techniques ${ }^{49}$. Ejection fraction is commonly preserved in patients with cardiac amyloidosis until late in the disease, however stroke volume is frequently impaired at an earlier stage ${ }^{49}$. Furthermore, the typical relative apical sparing on strain imaging has both diagnostic and prognostic values ${ }^{55}$. This typical pattern is independently predictive of all-cause mortality or the need for heart transplantation in patients with cardiac amyloidosis. Patients with a low ejection fraction and typical apical sparing were found to have the worst prognosis ${ }^{55}$.

\section{[H3] Prognostic data from tissue characterisation findings}

Numerous surrogate markers of amyloid burden such as uptake grade by bone scintigraphy and the transmurality of LGE and ECV by CMR are valuable predictors of prognosis in both types of cardiac amyloidosis. However, the prognostic role of the markers of myocardial oedema, native $\mathrm{T} 1$ and $\mathrm{T} 2$, are less straightforward ${ }^{79,133}$. Native $\mathrm{T} 1$ and $\mathrm{T} 2$ are independent prognostic markers in AL-CM, but not ATTR-CM, probably owing to the fact that myocardial amyloid infiltration is not the only mechanism involved in the two cardiomyopathes, but myocardial toxicity has an important role in disease pathophysiology and progression ${ }^{84}$. Bone scintigraphy results might also have prognostic implications, with a grade of 2 and 3 being associated with worse outcomes compared with a grade of $0^{134}$. A $\mathrm{H} / \mathrm{CL}$ ratio of $\geq 1.6$ is also associated with worse outcomes ${ }^{93}$.

\section{[H2] Monitoring cardiac amyloidosis with imaging}

At present, the serum concentration of NT-proBNP and echocardiographic measures are the reference standard for assessing cardiac responses to treatment in cardiac amyloidosis ${ }^{135}$, however, neither parameter directly quantifies amyloid burden ${ }^{82}$. Both NT-proBNP levels and myocardial strain represent processes that are downstream of amyloid deposition, NTproBNP levels are often confounded by renal impairment ${ }^{136}$ and myocardial strain is not well 
standardized and can be influenced by changes in preload and afterload ${ }^{137}$. CMR is a sensitive tool for characterizing myocardial amyloid deposits, and given that it can potentially dissect the different processes occurring in the myocardium (such as amyloid infiltration, cardiomyocyte response and myocardial edema), CMR seems to be ideal for tracking these changes in response to treatment (Figure 4). CMR has been shown to demonstrate progression or regression linked to clonal responses to therapy in AL amyloidosis in a retrospective study ${ }^{83}$, however, to date, no data on amyloid regression in ATTR-CM by imaging techniques has been reported.

\section{[H1] Future perspectives}

With several new drug therapies currently in development for both AL-CM and ATTR-CM, the potential of imaging in diagnosing and managing cardiac amyloidosis is greater than ever. Much like multiple myeloma, the treatment landscape of AL amyloidosis is rapidly expanding with the development of novel systemic therapies, and patients are living longer than ever before. Standard chemotherapy treatment has been replaced with newer drugs such as bortezomib, carfilzomib and ixazomib or daratumumab, lenalidomide and pomalidomide ${ }^{138}$. High-dose melphalan supported by autologous stem cell transplantation remains the therapeutic option for low-risk patients with $\mathrm{AL}$ amyloidosis ${ }^{3}$. The combination of (R)-1-[6-[(R)-2-carboxy-pyrrolidin-1-yl]-6-oxo-hexanoyl]pyrrolidine-2-carboxylic acid (also known as CPHPC) with anti-serum amyloid P component antibody was shown to eradicate amyloid deposits from the liver and spleen; additional studies to explore the cardiac response are planned ${ }^{139}$.

The ATTR-ACT study ${ }^{24}$ demonstrated that tafamidis, a transthyretin stabilizer, improved survival, reduced cardiovascular hospitalizations and preserved quality of life and exercise tolerance for patients with ATTR-CM. Reductions in cardiovascular hospitalizations and mortality were only observed at 9 and 18 months after treatment, respectively. Additionally, patients with less severe disease derived the greatest benefits from tafamidis treatment. Other agents that inhibit the production of transthyretin (via RNA interference) have subsequently been shown to be effective in patients with ATTRm and peripheral neuropathy ${ }^{21,22}$ and are currently being tested in patients with ATTR-CM. Given that all these therapies are remarkably expensive, optimization of their use is critical. Imaging techniques are paramount in the early detection of cardiac amyloidosis, when therapy is most successful, and are 
important in the assessment of treatment response, guiding the clinician on when to escalate the dose, whether to switch or combine therapies, and when to stop treatment owing to success or failure. Cardiac imaging can also potentially inform on the treatment responses in the different disease subclasses, with the ultimate aim of providing individualized multimodal treatment in the clinic.

\section{[H1] Conclusions}

Cardiac amyloidosis is an increasingly recognised systemic disease with diverse manifestations. A high index of suspicion is essential for the recognition of this condition at an early stage so that effective treatment can be initiated and outcomes potentially improved. Advanced imaging techniques including echocardiography, CMR and nuclear imaging are pivotal for the diagnostic and prognostic assessment of cardiac amyloidosis. These techniques should frequently be used in concert to maximize diagnostic and prognostic capacity and frequently obviate the need for endomyocardial biopsy, although complementary assessments such as family screening and genetic studies might be appropriate. Endomyocardial biopsy is the gold standard and is advised for the diagnosis of AL-CM. Endomyocardial biopsy is also advised for the diagnosis of full length amyloid fibre ATTR-CM or in light of equivocal bone scintigraphy results. The use of these powerful diagnostic imaging tools, notably CMR and nuclear techniques, for the identification and management of cardiac amyloidosis facilitates major improvements in the management of this life-limiting condition, driven by the development of novel therapies that are changing the course of disease.

\section{Acknowledgements}

M.F. is supported by a British Heart Foundation Intermediate Clinical Research Fellowship (FS/18/21/33447). We thank our many physician colleagues for referring and caring for the patients.

\section{Competing interests statement}

The authors have no competing interests to declare. 


\section{References:}

1 Falk, R. H., Kruger, J. \& Quarta, C. C. Senile Systemic Amyloidosis Is a Markedly Underdiagnosed Cardiomyopathy: Experience of a Cardiac Amyloidosis Program. Journal of the American College of Cardiology 61, E1241-E1241, doi:Doi 10.1016/S07351097(13)61241-3 (2013).

2 Maurer, M. S., Elliott, P., Comenzo, R., Semigran, M. \& Rapezzi, C. Addressing Common Questions Encountered in the Diagnosis and Management of Cardiac Amyloidosis. Circulation 135, 1357-1377, doi:10.1161/Circulationaha.116.024438 (2017).

3 Wechalekar, A. D., Gillmore, J. D. \& Hawkins, P. N. Systemic amyloidosis. Lancet 387, 2641-2654, doi:10.1016/S0140-6736(15)01274-X (2016).

4 Pinney, J. H. et al. Systemic amyloidosis in England: an epidemiological study. $\mathrm{Br} J$ Haematol 161, 525-532 (2013).

5 Gertz, M. A., Lacy, M. Q., Dispenzieri, A. \& Hayman, S. R. Amyloidosis. Best Pract Res Cl Ha 18, 709-727, doi:10.1016/j.beha.2005.01.030 (2005).

6 Merlini, G. CyBorD: stellar response rates in AL amyloidosis. Blood 119, 4343-4345, doi:10.1182/blood-2012-03-413112 (2012).

$7 \quad$ Falk, R. H., Alexander, K. M., Liao, R. \& Dorbala, S. AL (Light-Chain) Cardiac Amyloidosis: A Review of Diagnosis and Therapy. J Am Coll Cardiol 68, 1323-1341, doi:10.1016/j.jacc.2016.06.053 (2016).

8 Muchtar, E. et al. Improved outcomes for newly diagnosed AL amyloidosis between 2000 and 2014: cracking the glass ceiling of early death. Blood 129, 2111-2119, doi:10.1182/blood-2016-11-751628 (2017).

9 Jaccard, A. et al. Efficacy of bortezomib, cyclophosphamide and dexamethasone in treatmentnaive patients with high-risk cardiac AL amyloidosis (Mayo Clinic stage III). Haematologica 99, 1479-1485, doi:10.3324/haematol.2014.104109 (2014).

10 Mirzoyev, S. A. et al. Cardiac Amyloid Deposition is Common in Elderly Patients with Heart Failure and Preserved Ejection Fraction. Circulation 122 (2010).

11 Cornwell, G. G., Murdoch, W. L., Kyle, R. A., Westermark, P. \& Pitkanen, P. Frequency and Distribution of Senile Cardiovascular Amyloid - a Clinicopathologic Correlation. American Journal of Medicine 75, 618-623, doi:Doi 10.1016/0002-9343(83)90443-6 (1983).

12 Tanskanen, M. et al. Senile systemic amyloidosis affects $25 \%$ of the very aged and associates with genetic variation in alpha2-macroglobulin and tau: a population-based autopsy study. Ann Med 40, 232-239, doi:10.1080/07853890701842988 (2008).

13 Rapezzi, C. et al. Transthyretin-related amyloidoses and the heart: a clinical overview. Nat Rev Cardiol 7, 398-408, doi:10.1038/nrcardio.2010.67 (2010).

14 Connors, L. H., Lim, A., Prokaeva, T., Roskens, V. A. \& Costello, C. E. Tabulation of human transthyretin (TTR) variants, 2003. Amyloid 10, 160-184, doi:10.3109/13506120308998998 (2003).

15 Merlini, G. \& Westermark, P. The systemic amyloidoses: clearer understanding of the molecular mechanisms offers hope for more effective therapies. J Intern Med 255, 159-178 (2004).

16 Pinney, J. H. et al. Senile systemic amyloidosis: clinical features at presentation and outcome. J Am Heart Assoc 2, e000098, doi:10.1161/JAHA.113.000098 (2013).

17 Connors, L. H. et al. Cardiac amyloidosis in African Americans: comparison of clinical and laboratory features of transthyretin V122I amyloidosis and immunoglobulin light chain amyloidosis. Am Heart J 158, 607-614, doi:10.1016/j.ahj.2009.08.006 (2009).

18 Buxbaum, J. N. \& Ruberg, F. L. Transthyretin V122I ( pV142I)* cardiac amyloidosis: an agedependent autosomal dominant cardiomyopathy too common to be overlooked as a cause of significant heart disease in elderly African Americans. Genet Med 19, 733-742, doi:10.1038/gim.2016.200 (2017).

19 Rapezzi, C. et al. Systemic cardiac amyloidoses: disease profiles and clinical courses of the 3 main types. Circulation 120, 1203-1212, doi:10.1161/CIRCULATIONAHA.108.843334 (2009). 
20 Gillmore, J. D. et al. A new staging system for cardiac transthyretin amyloidosis. European Heart Journal 39, 2799-2806, doi:10.1093/eurheartj/ehxS89 (2018).

21 Adams, D. et al. Patisiran, an RNAi Therapeutic, for Hereditary Transthyretin Amyloidosis. New Engl J Med 379, 11-21, doi:10.1056/NEJMoa1716153 (2018).

22 Benson, M. D. et al. Inotersen Treatment for Patients with Hereditary Transthyretin Amyloidosis. New Engl J Med 379, 22-31, doi:10.1056/NEJMoa1716793 (2018).

23 Berk, J. L. et al. Repurposing Diflunisal for Familial Amyloid Polyneuropathy A Randomized Clinical Trial. Jama-J Am Med Assoc 310, 2658-2667, doi:10.1001/jama.2013.283815 (2013).

24 Maurer, M. S. et al. Tafamidis Treatment for Patients with Transthyretin Amyloid Cardiomyopathy. New England Journal of Medicine 379, 1007-1016, doi:10.1056/NEJMoa1805689 (2018).

25 Lane, T. et al. Natural History, Quality of Life, and Outcome in Cardiac Transthyretin Amyloidosis. Circulation 140, 16-26, doi:10.1161/CIRCULATIONAHA.118.038169 (2019).

26 Martinez-Naharro, A., Hawkins, P. N. \& Fontana, M. Cardiac amyloidosis. Clin Med (Lond) 18, s30-s35, doi:10.7861/clinmedicine.18-2-s30 (2018).

27 Longhi, S. et al. Atrial fibrillation in amyloidotic cardiomyopathy: prevalence, incidence, risk factors and prognostic role. Amyloid 22, 147-155, doi:10.3109/13506129.2015.1028616 (2015).

28 Mints, Y. Y., Doros, G., Berk, J. L., Connors, L. H. \& Ruberg, F. L. Features of atrial fibrillation in wild-type transthyretin cardiac amyloidosis: a systematic review and clinical experience. ESC Heart Fail 5, 772-779, doi:10.1002/ehf2.12308 (2018).

29 Martinez-Naharro, A. et al. High Prevalence of Intracardiac Thrombi in Cardiac Amyloidosis. J Am Coll Cardiol 73, 1733-1734, doi:10.1016/j.jacc.2019.01.035 (2019).

30 Feng, D. et al. Intracardiac thrombosis and anticoagulation therapy in cardiac amyloidosis. Circulation 119, 2490-2497, doi:10.1161/CIRCULATIONAHA.108.785014 (2009).

31 Gillmore, J. D. et al. A new staging system for cardiac transthyretin amyloidosis. Eur Heart J 39, 2799-2806, doi:10.1093/eurheartj/ehx589 (2018).

32 Grogan, M. et al. Natural History of Wild-Type Transthyretin Cardiac Amyloidosis and Risk Stratification Using a Novel Staging System. J Am Coll Cardiol 68, 1014-1020, doi:10.1016/j.jacc.2016.06.033 (2016).

33 Comenzo, R. L. et al. Consensus guidelines for the conduct and reporting of clinical trials in systemic light-chain amyloidosis. Leukemia 26, 2317-2325, doi:10.1038/leu.2012.100 (2012).

34 Sperry, B. W. et al. Are classic predictors of voltage valid in cardiac amyloidosis? A contemporary analysis of electrocardiographic findings. Int J Cardiol 214, 477-481, doi:10.1016/j.ijcard.2016.04.030 (2016).

35 Dubrey, S. W. et al. The clinical features of immunoglobulin light-chain (AL) amyloidosis with heart involvement. Qjm-Mon J Assoc Phys 91, 141-157, doi:DOI 10.1093/qjmed/91.2.141 (1998).

36 Murtagh, B. et al. Electrocardiographic findings in primary systemic amyloidosis and biopsyproven cardiac involvement. American Journal of Cardiology 95, 535-537, doi:10.1016/j.amjcard.2004.10.028 (2005).

37 Habib, G. et al. Multimodality Imaging in Restrictive Cardiomyopathies: An EACVI expert consensus document In collaboration with the "Working Group on myocardial and pericardial diseases" of the European Society of Cardiology Endorsed by The Indian Academy of Echocardiography. Eur Heart J Cardiovasc Imaging 18, 1090-1121, doi:10.1093/ehjci/jex034 (2017).

38 Ruberg, F. L., Grogan, M., Hanna, M., Kelly, J. W. \& Maurer, M. S. Transthyretin Amyloid Cardiomyopathy: JACC State-of-the-Art Review. J Am Coll Cardiol 73, 2872-2891, doi:10.1016/j.jacc.2019.04.003 (2019).

39 Sperry, B. et al. Are classic predictors of voltage valid in cardiac amyloidosis? A contemporary analysis of electrocardiographic findings. International Journal of Cardiology 214, 478-482, doi:10.1016/j.ijcard.2016.04.030 (2016). 
40 Cyrille, N. B., Goldsmith, J., Alvarez, J. \& Maurer, M. S. Prevalence and Prognostic Significance of Low QRS Voltage Among the Three Main Types of Cardiac Amyloidosis. American Journal of Cardiology 114, 1089-1093, doi:10.1016/j.amjcard.2014.07.026 (2014).

41 Dungu, J. et al. The electrocardiographic features associated with cardiac amyloidosis of variant transthyretin isoleucine 122 type in Afro-Caribbean patients. Am Heart J 164, 72-79, doi:10.1016/j.ahj.2012.04.013 (2012).

42 Fontana, M. et al. Differential Myocyte Responses in Patients with Cardiac Transthyretin Amyloidosis and Light-Chain Amyloidosis: A Cardiac MR Imaging Study. Radiology 277, 388-397, doi:10.1148/radiol.2015141744 (2015).

43 Martinez-Naharro, A. et al. Native T1 and Extracellular Volume in Transthyretin Amyloidosis. JACC Cardiovasc Imaging 12, 810-819, doi:10.1016/j.jcmg.2018.02.006 (2019).

44 Hongo, M. et al. Comparison of electrocardiographic findings in patients with AL (primary) amyloidosis and in familial amyloid polyneuropathy and anginal pain and their relation to histopathologic findings. Am J Cardiol 85, 849-853, doi:10.1016/s0002-9149(99)00879-6 (2000).

45 Pozo, E. et al. Cardiac magnetic resonance evaluation of left ventricular remodelling distribution in cardiac amyloidosis. Heart 100, 1688-1695, doi:10.1136/heartjnl-2014-305710 (2014).

46 Falk, R. H. Diagnosis and management of the cardiac amyloidoses. Circulation 112, 20472060, doi:10.1161/CIRCULATIONAHA.104.489187 (2005).

47 Gertz, M. A. et al. Definition of organ involvement and treatment response in immunoglobulin light chain amyloidosis (AL): a consensus opinion from the 10th International Symposium on Amyloid and Amyloidosis, Tours, France, 18-22 April 2004. Am J Hematol 79, 319-328, doi:10.1002/ajh.20381 (2005).

48 Austin, B. A. et al. Comparison of functional status, electrocardiographic, and echocardiographic parameters to mortality in endomyocardial-biopsy proven cardiac amyloidosis. Am J Cardiol 103, 1429-1433, doi:10.1016/j.amjcard.2009.01.361 (2009).

49 Knight, D. S. et al. Cardiac Structural and Functional Consequences of Amyloid Deposition by Cardiac Magnetic Resonance and Echocardiography and Their Prognostic Roles. JACC Cardiovasc Imaging 12, 823-833, doi:10.1016/j.jcmg.2018.02.016 (2019).

50 Tsang, W. \& Lang, R. M. Echocardiographic evaluation of cardiac amyloid. Curr Cardiol Rep 12, 272-276, doi:10.1007/s11886-010-0108-7 (2010).

51 Klein, A. L. et al. Doppler characterization of left ventricular diastolic function in cardiac amyloidosis. J Am Coll Cardiol 13, 1017-1026, doi:10.1016/0735-1097(89)90254-4 (1989).

52 Pagourelias, E. D. et al. Echo Parameters for Differential Diagnosis in Cardiac Amyloidosis: A Head-to-Head Comparison of Deformation and Nondeformation Parameters. Circulation. Cardiovascular imaging 10, e005588, doi:10.1161/CIRCIMAGING.116.005588 (2017).

53 Pewsner, D. et al. Ruling a diagnosis in or out with "SpPIn" and "SnNOut": a note of caution. BMJ 329, 209-213, doi:10.1136/bmj.329.7459.209 (2004).

54 Phelan, D. et al. Relative apical sparing of longitudinal strain using two-dimensional speckletracking echocardiography is both sensitive and specific for the diagnosis of cardiac amyloidosis. Heart 98, 1442-1448, doi:10.1136/heartjnl-2012-302353 (2012).

55 Senapati, A. et al. Prognostic implication of relative regional strain ratio in cardiac amyloidosis. Heart 102, 748-754, doi:10.1136/heartjnl-2015-308657 (2016).

56 Rapezzi, C. \& Fontana, M. Relative Left Ventricular Apical Sparing of Longitudinal Strain in Cardiac Amyloidosis Is it Just Amyloid Infiltration? Jacc-Cardiovasc Imag 12, 1174-1176, doi:10.1016/j.jcmg.2018.07.007 (2019).

57 Rapezzi, C. \& Fontana, M. Relative Left Ventricular Apical Sparing of Longitudinal Strain in Cardiac Amyloidosis: Is it Just Amyloid Infiltration? JACC Cardiovasc Imaging 12, 11741176, doi:10.1016/j.jcmg.2018.07.007 (2019).

58 Banypersad, S. M. The Evolving Role of Cardiovascular Magnetic Resonance Imaging in the Evaluation of Systemic Amyloidosis. Magn Reson Insights 12, 1178623X19843519, doi:10.1177/1178623X19843519 (2019). 
59 Pennell, D. J. Cardiovascular magnetic resonance: twenty-first century solutions in cardiology. Clin Med (Lond) 3, 273-278, doi:10.7861/clinmedicine.3-3-273 (2003).

60 Martinez-Naharro, A. et al. Magnetic Resonance in Transthyretin Cardiac Amyloidosis. J Am Coll Cardiol 70, 466-477, doi:10.1016/j.jacc.2017.05.053 (2017).

61 White, S. K. et al. T1 Mapping for Myocardial Extracellular Volume Measurement by CMR Bolus Only Versus Primed Infusion Technique. Jacc-Cardiovasc Imag 6, 955-962, doi:10.1016/j.jcmg.2013.01.011 (2013).

62 Reiter, T. et al. Minimizing risk of nephrogenic systemic fibrosis in cardiovascular magnetic resonance. J Cardiovasc Magn Reson 14, 31, doi:10.1186/1532-429X-14-31 (2012).

63 Koenig, S. H., Spiller, M., Brown, R. D., 3rd \& Wolf, G. L. Relaxation of water protons in the intra- and extracellular regions of blood containing Gd(DTPA). Magn Reson Med 3, 791795 (1986).

64 Kim, R. J. et al. Relationship of elevated 23Na magnetic resonance image intensity to infarct size after acute reperfused myocardial infarction. Circulation 100, 185-192, doi:10.1161/01.cir.100.2.185 (1999).

65 Rehwald, W. G., Fieno, D. S., Chen, E. L., Kim, R. J. \& Judd, R. M. Myocardial magnetic resonance imaging contrast agent concentrations after reversible and irreversible ischemic injury. Circulation 105, 224-229, doi:10.1161/hc0202.102016 (2002).

66 Maceira, A. M. et al. Cardiovascular magnetic resonance in cardiac amyloidosis. Circulation 111, 186-193, doi:10.1161/01.CIR.0000152819.97857.9D (2005).

67 Fontana, M., Chung, R., Hawkins, P. N. \& Moon, J. C. Cardiovascular magnetic resonance for amyloidosis. Heart failure reviews 20, 133-144, doi:10.1007/s10741-014-9470-7 (2015).

68 Kwong, R. Y. \& Falk, R. H. Cardiovascular magnetic resonance in cardiac amyloidosis. Circulation 111, 122-124, doi:10.1161/01.CIR.0000153623.02240.20 (2005).

69 Fontana, M. et al. A case report in cardiovascular magnetic resonance: the contrast agent matters in amyloid. BMC Med Imaging 17, 3, doi:10.1186/s12880-016-0173-5 (2017).

70 Moon, J. C. et al. Myocardial T1 mapping and extracellular volume quantification: a Society for Cardiovascular Magnetic Resonance (SCMR) and CMR Working Group of the European Society of Cardiology consensus statement. J Cardiovasc Magn Reson 15, 92, doi:10.1186/1532-429X-15-92 (2013).

71 Kellman, P., Arai, A. E., McVeigh, E. R. \& Aletras, A. H. Phase-sensitive inversion recovery for detecting myocardial infarction using gadolinium-delayed hyperenhancement. Magn Reson Med 47, 372-383 (2002).

72 Fontana, M. et al. Prognostic Value of Late Gadolinium Enhancement Cardiovascular Magnetic Resonance in Cardiac Amyloidosis. Circulation 132, 1570-1579, doi:10.1161/CIRCULATIONAHA.115.016567 (2015).

73 Bollee, G. et al. Presentation and outcome of patients with systemic amyloidosis undergoing dialysis. Clin J Am Soc Nephrol 3, 375-381, doi:10.2215/CJN.02470607 (2008).

74 Lachmann, H. J. \& Gillmore, J. D. Renal amyloidosis. Br J Hosp Med (Lond) 71, 83-86, doi:10.12968/hmed.2010.71.2.46485 (2010).

75 Fontana, M. et al. Native T1 mapping in transthyretin amyloidosis. JACC Cardiovasc Imaging 7, 157-165, doi:10.1016/j.jcmg.2013.10.008 (2014).

76 Karamitsos, T. D. et al. Noncontrast T1 mapping for the diagnosis of cardiac amyloidosis. JACC Cardiovasc Imaging 6, 488-497, doi:10.1016/j.jcmg.2012.11.013 (2013).

77 Sado, D. M. et al. Identification and assessment of Anderson-Fabry disease by cardiovascular magnetic resonance noncontrast myocardial T1 mapping. Circ Cardiovasc Imaging 6, 392398, doi:10.1161/CIRCIMAGING.112.000070 (2013).

78 Baggiano, A. et al. Noncontrast Magnetic Resonance for the Diagnosis of Cardiac Amyloidosis. JACC Cardiovasc Imaging, doi:10.1016/j.jcmg.2019.03.026 (2019).

79 Banypersad, S. M. et al. T1 mapping and survival in systemic light-chain amyloidosis. Eur Heart J 36, 244-251, doi:10.1093/eurheartj/ehu444 (2015).

80 Fontana, M. et al. Differential Myocyte Responses in Patients with Cardiac Transthyretin Amyloidosis and Light-Chain Amyloidosis: A Cardiac MR Imaging Study. Radiology 277, 388-397, doi:10.1148/radiol.2015141744 (2015). 
81 Martinez-Naharro, A. et al. Native T1 and Extracellular Volume in Transthyretin Amyloidosis. Jacc-Cardiovasc Imag 12, 810-819, doi:10.1016/j.jcmg.2018.02.006 (2019). Banypersad, S. M. et al. Quantification of myocardial extracellular volume fraction in systemic AL amyloidosis: an equilibrium contrast cardiovascular magnetic resonance study. Circulation. Cardiovascular imaging 6, 34-39 (2013).

83 Martinez-Naharro, A. et al. CMR-Verified Regression of Cardiac AL Amyloid After Chemotherapy. JACC Cardiovasc Imaging 11, 152-154, doi:10.1016/j.jcmg.2017.02.012 (2018).

84 Kotecha, T. et al. Myocardial Edema and Prognosis in Amyloidosis. J Am Coll Cardiol 71, 2919-2931, doi:10.1016/j.jacc.2018.03.536 (2018).

85 Kellman, P. et al. Myocardial perfusion cardiovascular magnetic resonance: optimized dual sequence and reconstruction for quantification. J Cardiovasc Magn Reson 19, 43, doi:10.1186/s12968-017-0355-5 (2017).

86 Shirahama, T. \& Cohen, A. S. High-resolution electron microscopic analysis of the amyloid fibril. J Cell Biol 33, 679-708, doi:10.1083/jcb.33.3.679 (1967).

87 Nordlinger, M., Magnani, B., Skinner, M. \& Falk, R. H. Is elevated plasma B-natriuretic peptide in amyloidosis simply a function of the presence of heart failure? Am J Cardiol 96, 982-984, doi:10.1016/j.amjcard.2005.05.057 (2005).

88 Martinez-Naharro, A. et al. Routine Identification of Hypoperfusion in Cardiac Amyloidosis by Myocardial Blood Flow Mapping. Heart 103, A24-A25, doi:10.1136/heartjnl-2017311399.28 (2017).

89 Wizenberg, T. A., Muz, J., Sohn, Y. H., Samlowski, W. \& Weissler, A. M. Value of positive myocardial technetium-99m-pyrophosphate scintigraphy in the noninvasive diagnosis of cardiac amyloidosis. Am Heart J 103, 468-473, doi:10.1016/0002-8703(82)90331-3 (1982). Puille, M. et al. $99 \mathrm{mTc}$-DPD scintigraphy in transthyretin-related familial amyloidotic polyneuropathy. Eur J Nucl Med Mol Imaging 29, 376-379 (2002).

91 Bokhari, S., Shahzad, R., Castano, A. \& Maurer, M. S. Nuclear imaging modalities for cardiac amyloidosis. Journal of Nuclear Cardiology 21, 175-184, doi:10.1007/s12350-0139803-2 (2014).

92 Bokhari, S. et al. (99m)Tc-pyrophosphate scintigraphy for differentiating light-chain cardiac amyloidosis from the transthyretin-related familial and senile cardiac amyloidoses.

Circulation. Cardiovascular imaging 6, 195-201, doi:10.1161/CIRCIMAGING.112.000132 (2013).

93 Castano, A. et al. Multicenter Study of Planar Technetium 99m Pyrophosphate Cardiac Imaging: Predicting Survival for Patients With ATTR Cardiac Amyloidosis. JAMA cardiology 1, 880-889, doi:10.1001/jamacardio.2016.2839 (2016).

94 Perugini, E. et al. Noninvasive etiologic diagnosis of cardiac amyloidosis using 99mTc-3,3diphosphono-1,2-propanodicarboxylic acid scintigraphy. J Am Coll Cardiol 46, 1076-1084, doi:10.1016/j.jacc.2005.05.073 (2005).

95 Rapezzi, C. et al. Role of $(99 \mathrm{~m})$ Tc-DPD scintigraphy in diagnosis and prognosis of hereditary transthyretin-related cardiac amyloidosis. JACC Cardiovasc Imaging 4, 659-670, doi:10.1016/j.jcmg.2011.03.016 (2011).

96 Papantoniou, V. et al. Imaging of cardiac amyloidosis by $(99 \mathrm{~m}) \mathrm{Tc}-\mathrm{PYP}$ scintigraphy. Hell $J$ Nucl Med 18 Suppl 1, 42-50 (2015).

97 Galat, A. et al. Usefulness of (99m)Tc-HMDP scintigraphy for the etiologic diagnosis and prognosis of cardiac amyloidosis. Amyloid 22, 210-220, doi:10.3109/13506129.2015.1072089 (2015).

98 Glaudemans, A. W. et al. Bone scintigraphy with (99m)technetium-hydroxymethylene diphosphonate allows early diagnosis of cardiac involvement in patients with transthyretinderived systemic amyloidosis. Amyloid 21, 35-44 (2014).

99 Rapezzi, C., Gagliardi, C. \& Milandri, A. Analogies and disparities among scintigraphic bone tracers in the diagnosis of cardiac and non-cardiac ATTR amyloidosis. J Nucl Cardiol, doi:10.1007/s12350-018-1235-6 (2018). 
100 Pilebro, B. et al. (99m)Tc-DPD uptake reflects amyloid fibril composition in hereditary transthyretin amyloidosis. Ups J Med Sci 121, 17-24, doi:10.3109/03009734.2015.1122687 (2016).

101 Stats, M. A. \& Stone, J. R. Varying levels of small microcalcifications and macrophages in ATTR and AL cardiac amyloidosis: implications for utilizing nuclear medicine studies to subtype amyloidosis. Cardiovasc Pathol 25, 413-417, doi:10.1016/j.carpath.2016.07.001 (2016).

102 Falk, R. H., Lee, V. W., Rubinow, A., Hood, W. B., Jr. \& Cohen, A. S. Sensitivity of technetium-99m-pyrophosphate scintigraphy in diagnosing cardiac amyloidosis. Am J Cardiol 51, 826-830, doi:10.1016/s0002-9149(83)80140-4 (1983).

103 Hutt, D. F. et al. Utility and limitations of 3,3-diphosphono-1,2-propanodicarboxylic acid scintigraphy in systemic amyloidosis. Eur Heart J Cardiovasc Imaging 15, 1289-1298, doi:10.1093/ehjci/jeu107 (2014).

104 Gillmore, J. D. et al. Nonbiopsy Diagnosis of Cardiac Transthyretin Amyloidosis. Circulation 133, 2404-2412 (2016).

105 Vandenberghe, R. et al. 18F-flutemetamol amyloid imaging in Alzheimer disease and mild cognitive impairment: a phase 2 trial. Ann Neurol 68, 319-329, doi:10.1002/ana.22068 (2010).

106 Bateman, R. J. et al. Clinical and biomarker changes in dominantly inherited Alzheimer's disease. $N$ Engl J Med 367, 795-804, doi:10.1056/NEJMoa1202753 (2012).

107 Dorbala, S. et al. Imaging cardiac amyloidosis: a pilot study using (1)(8)F-florbetapir positron emission tomography. Eur J Nucl Med Mol Imaging 41, 1652-1662, doi:10.1007/s00259-0142787-6 (2014).

108 Osborne, D. R., Acuff, S. N., Stuckey, A. \& Wall, J. S. A Routine PET/CT Protocol with Streamlined Calculations for Assessing Cardiac Amyloidosis Using (18)F-Florbetapir. Front Cardiovasc Med 2, 23, doi:10.3389/fcvm.2015.00023 (2015).

109 Antoni, G. et al. In vivo visualization of amyloid deposits in the heart with 11C-PIB and PET. J Nucl Med 54, 213-220, doi:10.2967/jnumed.111.102053 (2013).

110 Lee, S. P. et al. 11C-Pittsburgh B PET imaging in cardiac amyloidosis. JACC Cardiovasc Imaging 8, 50-59 (2015).

111 Law, W. P., Wang, W. Y., Moore, P. T., Mollee, P. N. \& Ng, A. C. Cardiac Amyloid Imaging with 18F-Florbetaben PET: A Pilot Study. J Nucl Med 57, 1733-1739, doi:10.2967/jnumed.115.169870 (2016).

112 Paulus, W. J. et al. How to diagnose diastolic heart failure: a consensus statement on the diagnosis of heart failure with normal left ventricular ejection fraction by the Heart Failure and Echocardiography Associations of the European Society of Cardiology. Eur Heart $J \mathbf{2 8}$, 2539-2550, doi:10.1093/eurheartj/ehm037 (2007).

113 Komajda, M. \& Lam, C. S. Heart failure with preserved ejection fraction: a clinical dilemma. Eur Heart J 35, 1022-1032, doi:10.1093/eurheartj/ehu067 (2014).

114 Gonzalez-Lopez, E. et al. Wild-type transthyretin amyloidosis as a cause of heart failure with preserved ejection fraction. Eur Heart J 36, 2585-2594, doi:10.1093/eurheartj/ehv338 (2015).

115 Treibel, T. A. et al. Occult Transthyretin Cardiac Amyloid in Severe Calcific Aortic Stenosis: Prevalence and Prognosis in Patients Undergoing Surgical Aortic Valve Replacement. Circulation. Cardiovascular imaging 9, doi:10.1161/circimaging.116.005066 (2016).

116 Longhi, S. et al. Identification of TTR-related subclinical amyloidosis with 99mTc-DPD scintigraphy. JACC Cardiovasc Imaging 7, 531-532 (2014).

117 Cavalcante, J. L. et al. Cardiac amyloidosis is prevalent in older patients with aortic stenosis and carries worse prognosis. J Cardiovasc Magn Reson 19, 98, doi:10.1186/s12968-0170415-x (2017).

118 Castano, A. et al. Unveiling transthyretin cardiac amyloidosis and its predictors among elderly patients with severe aortic stenosis undergoing transcatheter aortic valve replacement. Eur Heart J 38, 2879-2887, doi:10.1093/eurheartj/ehx350 (2017).

119 Moon, J. C. et al. Gadolinium enhanced cardiovascular magnetic resonance in AndersonFabry disease. Evidence for a disease specific abnormality of the myocardial interstitium. Eur Heart J 24, 2151-2155, doi:10.1016/j.ehj.2003.09.017 (2003). 
120 Shah, K. B. et al. Transthyretin Cardiac Amyloidosis in Black Americans. Circ Heart Fail 9, e002558, doi:10.1161/CIRCHEARTFAILURE.115.002558 (2016).

121 Vogelsberg, H. et al. Cardiovascular magnetic resonance in clinically suspected cardiac amyloidosis: noninvasive imaging compared to endomyocardial biopsy. $J$ Am Coll Cardiol 51, 1022-1030, doi:10.1016/j.jacc.2007.10.049 (2008).

122 Austin, B. A. et al. Delayed hyper-enhancement magnetic resonance imaging provides incremental diagnostic and prognostic utility in suspected cardiac amyloidosis. JACC Cardiovasc Imaging 2, 1369-1377, doi:10.1016/j.jcmg.2009.08.008 (2009).

123 Ruberg, F. L. et al. Diagnostic and prognostic utility of cardiovascular magnetic resonance imaging in light-chain cardiac amyloidosis. Am J Cardiol 103, 544-549, doi:10.1016/j.amjcard.2008.09.105 (2009).

124 Syed, I. S. et al. Role of cardiac magnetic resonance imaging in the detection of cardiac amyloidosis. JACC Cardiovasc Imaging 3, 155-164, doi:10.1016/j.jcmg.2009.09.023 (2010).

125 Damy, T. et al. Prevalence and clinical phenotype of hereditary transthyretin amyloid cardiomyopathy in patients with increased left ventricular wall thickness. Eur Heart J 37, 1826-1834, doi:10.1093/eurheartj/ehv583 (2016).

126 Sperry, B. W. et al. Tenosynovial and Cardiac Amyloidosis in Patients Undergoing Carpal Tunnel Release. J Am Coll Cardiol 72, 2040-2050, doi:10.1016/j.jacc.2018.07.092 (2018).

127 Fosbol, E. L. et al. Association of Carpal Tunnel Syndrome With Amyloidosis, Heart Failure, and Adverse Cardiovascular Outcomes. J Am Coll Cardiol 74, 15-23, doi:10.1016/j.jacc.2019.04.054 (2019).

128 Dispenzieri, A. et al. Superior survival in primary systemic amyloidosis patients undergoing peripheral blood stem cell transplantation: a case-control study. Blood 103, 3960-3963, doi:10.1182/blood-2003-12-4192 (2004).

129 Mohty, D. et al. Left atrial size is an independent predictor of overall survival in patients with primary systemic amyloidosis. Arch Cardiovasc Dis 104, 611-618, doi:10.1016/j.acvd.2011.10.004 (2011).

130 Klein, A. L. et al. Prognostic significance of Doppler measures of diastolic function in cardiac amyloidosis. A Doppler echocardiography study. Circulation 83, 808-816, doi:10.1161/01.cir.83.3.808 (1991).

131 Koyama, J. \& Falk, R. H. Prognostic significance of strain Doppler imaging in light-chain amyloidosis. JACC Cardiovasc Imaging 3, 333-342, doi:10.1016/j.jcmg.2009.11.013 (2010).

132 Siepen, F. A. D. et al. Predictors of survival stratification in patients with wild-type cardiac amyloidosis. Clin Res Cardiol 107, 158-169, doi:10.1007/s00392-017-1167-1 (2018).

133 Kotecha, T., Martinez-Naharro, A., Gillmore, J. D., Hawkins, P. N. \& Fontana, M. Reply: Amyloidosis in the Era of Mass Spectrometry-Based Proteomics. J Am Coll Cardiol 72, 1881, doi:10.1016/j.jacc.2018.08.1036 (2018).

134 Hutt, D. F. et al. Prognostic utility of the Perugini grading of 99mTc-DPD scintigraphy in transthyretin (ATTR) amyloidosis and its relationship with skeletal muscle and soft tissue amyloid. Eur Heart J Cardiovasc Imaging 18, 1344-1350, doi:10.1093/ehjci/jew325 (2017).

135 Dispenzieri, A. et al. Serum cardiac troponins and N-terminal pro-brain natriuretic peptide: a staging system for primary systemic amyloidosis. J Clin Oncol 22, 3751-3757, doi:10.1200/JCO.2004.03.029 (2004).

136 Emdin, M. et al. Cardiac natriuretic hormones, neuro-hormones, thyroid hormones and cytokines in normal subjects and patients with heart failure. Clin Chem Lab Med 42, 627-636, doi:10.1515/CCLM.2004.108 (2004).

137 Clerico, A. \& Emdin, M. Diagnostic accuracy and prognostic relevance of the measurement of cardiac natriuretic peptides: a review. Clin Chem 50, 33-50, doi:10.1373/clinchem.2003.024760 (2004).

138 Rysava, R. AL amyloidosis: advances in diagnostics and treatment. Nephrol Dial Transplant, doi:10.1093/ndt/gfy291 (2018).

139 Richards, D. B. et al. Therapeutic Clearance of Amyloid by Antibodies to Serum Amyloid P Component. N Engl J Med 373, 1106-1114 (2015). 


\section{Key points}

- Cardiac amyloidosis is a life-threatening and progressive cause of heart failure that is often underdiagnosed or misdiagnosed

- Early and accurate diagnosis of cardiac amyloidosis is crucial for the implementation of appropriate patient care, and is now more important than ever given the availability of new therapies

- Certain clinical criteria have been established that warrant screening for cardiac amyloidosis

- Once cardiac amyloidosis is suspected, a definitive diagnosis can usually be achieved noninvasively through the use of imaging techniques such as echocardiography, bone scintigraphy and cardiac magnetic resonance

- The combination of advanced cardiac imaging modalities provides valuable prognostic information and is paramount for the monitoring of disease progression and treatment response 


\section{Box 1 | Red flags for cardiac amyloidosis}

Evidence of heart failure, in addition to:

- Preserved ejection fraction without hypertension

- Discrepancy between QRS voltage and left ventricular thickness

- Atrioventricular block in the presence of increased wall thickness

- Hypertrophic phenotype on echocardiography with typical 'bulls-eye' pattern on strain imaging

- Diffuse late gadolinium enhancement on cardiac magnetic resonance with subendocardial or transmural distribution

- Evidence of cardiac uptake on bone scintigraphy

- Presence of bilateral carpal tunnel syndrome

- Intolerance of previously used cardiovascular medications

- Symptoms of neuropathy or autonomic dysfunction

- Mildly increased troponin levels on repeated tests 
Table 1. Summary of the benefits of each technique in relation to the different stages of the diagnostic process in cardiac amyloidosis.

\begin{tabular}{|l|l|l|l|l|}
\hline \multicolumn{5}{|c|}{ AL amyloid cardiomyopathy } \\
\hline & Subclinical & Early & Established & Advanced \\
\hline Echocardiography & - & + & ++ & ++ \\
\hline CMR & + & ++ & +++ & +++ \\
\hline Bone scintigraphy & + & ++ & +++ & +++ \\
\hline \multicolumn{7}{|c|}{ ATTR amyloid cardiomyopathy } & Advanced \\
\hline & Subclinical & Early & Established & ++ \\
\hline Echocardography & - & + & ++ & +++ \\
\hline CMR & + & ++ & +++ & + \\
\hline Bone scintigraphy & + & + & + & \\
\hline
\end{tabular}

$\mathrm{CMR}$, cardiac magnetic resonance

\section{Figure legends}

Figure 1. Multidisciplinary workup of a patient with ATTR amyloid cardiomyopathy. Images show: a| Typical four chamber echocardiographic view and strain pattern characteristic of an infiltrative process. $\mathrm{b}$ | Four-chamber steady-state free-precession image corresponding 4 chamber native T1 map; corresponding LGE image showing transmural LGE and corresponding ECV map with high values $\mathrm{c} \mid$ Whole-body anterior ${ }^{99 \mathrm{~m}} \mathrm{Tc}-3,3$ diphosphono-1,2-propanodicarboxylic acid scintigraphy (left) and single-photon emission computerized tomography (right) showing Perugini grade 2 abnormal cardiac uptake.

Figure 2. CMR shows different pathophysiological mechanisms in cardiac amyloidosis. Elevation of native $\mathrm{T} 1$ and $\mathrm{T} 2$ values are commonly seen in myocardial oedema associated with cardiac amyloidosis. The rest perfusion state demonstrates low myocardial blood values, whereas a typical late gadolinium enhancement (LGE) pattern and elevated extracellular volume (ECV) values are markers of amyloid burden. CMR, cardiac magnetic resonance. 
Figure 3. CMR findings in left ventricular hypertrophy. Short axis balanced steady-state free-precession image, native T1, late gadolinium enhancement (LGE) and extracellular volume $(\mathrm{ECV})$ is shown in four different pathologies that cause left ventricular hypertrophy. Native T1 values are elevated in cardiac amyloidosis, hypertrophic cardiomyopathy (and hypertensive heart disease, but reduced in Anderson-Fabry disease. This pattern of LGE is characteristic of cardiac amyloidosis (diffuse subendocardial LGE, transmural at the basal septum), whereas patchy LGE in the septum is indicative of hypertrophic cardiomyopathy and mid-wall LGE in the basal inferolateral wall is suggestive of Anderson-Fabry disease. In hypertensive heart disease, only subtle patchy LGE in the septum can be observed. ECV values are characteristically and diffusely high in cardiac amyloidosis, but in the other pathologies are only elevated in the areas where there is LGE.

Figure 4. Regression of cardiac amyloidosis by CMR. Top row shows elevated native T1 values, typical subendocardial late gadolinium enhancement (LGE) pattern and elevated extracellular volume (ECV) values in a patient with AL amyloid cardiomyopathy before chemotherapy. After 1 year of chemotherapy, native T1 and ECV values are substantially reduced and the pattern of LGE has improved.

Figure 5. Whole body Perugini visual score of cardiac uptake on ${ }^{99 \mathrm{~m}} \mathrm{Tc}-3,3-$ diphosphono-1,2propanodicarboxylic acid scintigraphy. Grade 0 represents no cardiac uptake, grade 1 represents mild cardiac uptake (less than bone), grade 2 represents cardiac uptake greater than in bone, (but bone uptake still remains visible) and grade 3 represents substantial cardiac uptake with only a weak or no signal evident in bone. 